Résumé : Notre article a pour objet l'étude des relations entre la France et la Serbie durant la $I^{\circ}$ Guerre mondiale, à travers l'analyse des relations entre soldats français et serbes. Dès 1903 et la prise du pouvoir par les Karadjordjević, la politique extérieure serbe se réoriente vers la France en abandonnant les empires centraux des Obrenović. La France, elle, voit dans le Piémont serbe un allié intéressant dans son dispositif de contournement des Empires centraux. Dès les années 1900, la conjonction de ces deux facteurs explique la forte présence des entreprises et de l'Etat français en Serbie. A travers des projets d'infrastructures ou bancaires la France commence à s'implanter en Serbie. La I ${ }^{\circ}$ Guerre mondiale sera l'occasion d'une véritable ancrage de la France dans les Balkans et de la création de liens indéfectibles entre les peuples français et serbe. Les souffrances communes et la fraternité d'armes endurées sur le front de Salonique pendant trois ans (1916-1918) vont sceller fortement l'attachement du peuple serbe à la France. L'armée serbe, qui sera obligée de capituler face aux Empires centraux en octobre 1915, éprouvera une gratitude éternelle à l'Armée d'orient qui sauve les soldats serbes après la retraite d'Albanie à l'hiver 1915, guérit toute une armée sur l'île de Corfou au printemps 1916 , puis combat dans une unité remarquable jusqu'à la percée du Front de Salonique le 15 septembre 1918. La victoire du Dobro polje, grâce au Maréchal Franchet d'Esperey et au général Tranié, sera la première victoire décisive de l'Entente. L'état français organise sur le sol national pendant la même période des conférences sur la Serbie, des Journées franco-serbes dans toutes les écoles et la prise en charge de milliers d'enfants serbes. La France développe enfin pendant ces trois années des projets de la France à moyen terme dans cette région 
d'Europe orientale, notamment en Macédoine, qui permettent d'étendre une implantation durable en Europe orientale. Ces liens renforcés facilitent l'implantation de la France dans les Balkans dans les années d'après-guerre, à travers la création $d u$ «Royaume des Serbes, Croates et Slovènes» en décembre 1918. En choisissant la monarchie serbe des Karadjordjevic, en dessinant les frontières et en installant une administration centralisée, la France fait du «Royaume des Serbes, Croates et Slovènes» son principal allié en Europe orientale. Cela ouvre la voie dans les années d'après-guerre au renforcement des liens militaires, économiques et culturels entre la France et le royaume des Karadjordjević.

Mots-clé : Dynastie des Karadjordjević, Front de Salonique, Armée d'orient, influence de la France, Serbie

\section{La Serbie en 1914 :}

\section{un allié récent mais solide de la France}

\section{La Serbie dans le jeu des puissances}

\section{Liens politiques, culturels et militaires}

\section{France/Serbie (1900-1914)}

Les liens entre la France et la petite Serbie étaient à la fois très solides et ténus depuis le retour de la dynastie des Karadjordjević en Serbie en $1903^{1}$, mais ils dénotaient en même temps une profonde méconnaissance des Serbes et de la Serbie par les Français avant la $\mathrm{I}^{\circ}$ Guerre mondiale.

Le publiciste Pierre de Lanux a bien décrit en 1916 la totale méconnaissance des caractères ethniques de chaque peuple balkanique par les Français lorsque le conflit mondial commença. Ainsi, les Serbes étaient pris pour des « montagnards 》 et des « orientaux au caractère indolent et fuyant "; l'ambassadeur de France en poste à Belgrade entre 1907 et 1914 évoquera même leur « somnolence coutumière $»^{2}$. Or les Serbes habitaient des vallées et des collines, et on ne pouvait les considérer de caractère oriental, car ils étaient d'origine slave ${ }^{3}$. Par ailleurs, la presse française avait l'habitude de parler des Serbes, mais seulement en les présentant sous une seule facette et de manière parfois dédaigneuse. Selon des journaux comme le Temps ou l'Illustration, c'étaient des "éleveurs de porcs » aux mœurs orientales et violentes. On les confondait souvent avec les Bulgares et le coup d'Etat sanglant de 1903, où le dernier

1 Castellan, G. (1991) Histoire des Balkans, Fayard, Paris, pp. 326-331.

2 SHM, SS Z 35, dossier H3- Affaires serbes.

3 Colin, A. (1918) Pour la géographie de la Serbie, consulter le très didactique ouvrage du géographe serbe Jovan Cvijic, La péninsule balkanique. Géographie humaine, Paris. 


\section{ALEXIS TROUDE}

descendant de la dynastie Obrenović fut décapité, était resté dans les mémoires.

Dans ces journaux à grand tirage, on estimait peu la stratégie et l'organisation militaire des Serbes, mais on s'intéressait surtout à leur goût pour le chant et les poèmes épiques. Les Français, à la veille du conflit, avaient en fait peur des vélléités combattives des Serbes : lorsqu'Henri Barby, journaliste à l'Aurore, raconta les victoires serbes contre les Ottomans en 1913, les lecteurs réagirent en pensant que cela risquait d'entraîner la France dans une nouvelle guerre ${ }^{4}$. Les dirigeants eux, par contre, étaient très bien informés des réalités locales. L'ambassadeur de France à Belgrade, Robert Descos, avait déjà dit dans sa note du 10 septembre 1912, adressée à son Ministre, que " la défiance du Bulgare domine toujours en Serbie »; le 4 février 1913, il opposait ainsi «le Serbe, infiniment plus cultivé, mais plus mou que son voisin de l'Est » au Bulgare « âpre et violent, arbitraire et dominateur ». Déjà, l'ambassadeur de France à Belgrade remarquait les prétentions territoriales des Bulgares sur Kumanovo et Skoplje, et la volonté des Serbes d'avoir un « débouché sur la mer Egée » depuis que la Bosnie-Herzégovine annexée par l'Autriche en 1908 lui avait barré la route de l'Adriatique ${ }^{5}$.

Dans ce contexte, la France officielle se rapprocha de la Serbie, Piémont des Balkans. Le roi Pierre $\mathrm{I}^{\circ}$ Karadjordjević, arrivé sur le trône en 1903, avait mené une politique totalement favorable à la France, ce qui changea complètement la politique française dans les années 1900 et 1910. Ayant fréquenté l'école militaire de Saint-Cyr à la fin des années 1860 puis participé à la guerre contre la Prusse aux côtés de la France, Pierre $\mathrm{I}^{\circ}$ de Serbie était un monarque sur lequel la France pouvait s'appuyer ${ }^{6}$.

Bien avant ce rapprochement diplomatique franco-serbe, la culture française s'était implantée dans la principauté de Serbie au XIX ${ }^{\circ}$ siècle. Déjà en 1838 , les Français avaient aidé le prince Miloš Obrenović à établir une Constitution et la langue française était enseignée dans les trois lycées dès 1848 et à la faculté de Belgrade à partir de 1880. Mais le contact le plus important avec le milieu culturel français fut l'envoi de jeunes boursiers serbes en France à partir du milieu du XIX $^{\circ}$ siècle. Cela avait le double avantage de les arracher à l'influence germanique- de

4 Barby, H. (1913) Les victoires serbes, Paris: Belfond.

5 Pavlovic, V. (1988) Télégrammes de la Légation française de Belgrade, in: Témoignages français sur les Serbes et la Serbie, Belgrade: Narodna Knjiga, pp. 67-85.

6 Batakovic, D. (2005) Histoire du peuple serbe, L'Age d'Homme, Paris, pp. $185-188$. 
Vuk Karadžić à Nikola Pašić, les figures éminentes de la Serbie avaient fait leurs études en Autriche-Hongrie ou en Allemagneet à constituer un corps de diplomates et de fonctionnaires fidèles à la France. Ce qu'on a appelé les «Parisiens » eurent pour nom Jovan Marinović, Filip Khristić, ou Milan Janković. En 1889, sur 33 boursiers envoyés à l'étranger, 14 le furent à Paris. A partir du milieu du XIX ${ }^{\circ}$ siècle, ils formaient une élite intellectuelle francophile qui allait influer sur le rapprochement entre la Serbie et la France. La conséquence en fut la présence d'hommes d'Etat proches de la France : en poste durant la Première Guerre mondiale, le Ministre des Finances Momčilo Ninčić et les ambassadeurs à Londres et à Paris avaient fait leurs études à Paris ${ }^{7}$.

La colonie française à Belgrade n'était pas nombreuse au XIX ${ }^{\circ}$ siècle, mais certains de ses membres avaient laissé des traces dans la vie publique de la Serbie ; le capitaine Magnant avait essayé de rétablir, après la paix de Paris, le transport fluvial sur la Save et le Danube et, en le reliant à la ligne Marseille-Galatz, de faire sortir le commerce serbe de sa dépendance vis-à-vis de l'Autriche. Mais c'est surtout le capitaine de génie Hippolyte Mondain qui retiendra notre attention. Il fut envoyé dans une première mission à Belgrade pendant la guerre de Crimée (185355) puis, en 1861, il était nommé Ministre de la Guerre par le gouvernement serbe ${ }^{8}$. En l'espace de quelques années, il dressa un plan d'ensemble des routes et défenses de la Serbie, refit le programme de l'école d'artillerie et forma un certain nombre de cadres militaires. Surtout, Mondain créa une armée de partisans rapidement mobilisable, la milice nationale serbe. En bon connaisseur des Balkans, il avait déjà constaté dans les années 1880 que « les manoeuvres s'exécutent avec un entrain et un ensemble qu'on serait loin d'espérer de troupes irrégulières »; « le peuple serbe possède un goût inné pour les armes et des qualités nécessaires pour faire un peuple guerrier $»^{9}$.

\section{La Serbie comme clé stratégique des Balkans}

La Serbie représentait en 1914 un allié important du dispositif diplomatique français. Comme le dit en 1916 l'historien Victor Bérard, la Serbie constituait, dans les Balkans, l' « élément principal de notre politique face à l'expansionnisme

7 Pavlovic, V. (1990) L'influence culturelle de la France en Serbie à l'époque des Constitutionnalistes, in: Rapports franco-yougoslaves, Belgrade: Institut d'Histoire, pp. 103-111.

8 SHAT, 7 N 1573, Dossier « Attachés militaires-Missions en Serbie ».

9 Vuksanovic-Anic, D. (1990) Les missions militaires françaises en Serbie de 1853 à 1886 et la question de la milice nationale, in: Rapports francoyougoslaves, Institut d'Histoire, Belgrade, pp. 120-130. 
germanique ». Or ces craintes furent reprises, de façon répétitive et alarmée, par les officiers du $3^{\circ}$ bureau : ainsi le 7 octobre 1915 était souligné ce qui fut appelé le « plan allemand », c'està-dire « réaliser au travers du territoire serbe la continuité des échanges et des territoires autrichien, bulgare et turc ${ }^{10}$. En effet, les Allemands contrôlaient en 1914 à la fois l'axe VienneSofia-Istanbul par l'Orient-Express et la route de l'Orient par le Berlin-Bagdad-Bahn. Des rapports du $2^{\circ}$ bureau sur les Balkans ressortait en 1915 le souci principal de la France : empêcher les Allemands d'aller plus en avant dans leur contrôle des richesses du Moyen-Orient, " terre de convoitises économiques et de rêves d'influence mondiale " pour l'Allemagne ${ }^{11}$. Pétrole de mer Caspienne, mines de fer et de charbon d'Irak ainsi que richesses agricoles de Turquie étaient pointées du doigt par le $2^{\circ}$ bureau $^{12}$. Or les deux axes Budapest/Salonique et Vienne/ Istanbul traversaient la Serbie. Depuis la crise de l'annexion de la Bosnie-Herzégovine par les Autrichiens en 1908, la France craignait un contournement de la Serbie par l'ouest et le sud (alliés albanais), ce qui aurait amené un affaiblissement de ses positions dans la région.

Deuxième souci de la diplomatie française en 1914, comme le dit une note du $2^{\circ}$ bureau du 7 octobre 1915, « la Quadruple Entente sait en effet à peu près maintenant quels sont ses adversaires dans les Balkans, mais elle ne sait pas quels sont ses amis ». La Roumanie était dirigée par un " Hohenzollern qui a signé des traités avec tout le monde », car son but était de « ne marcher qu'au dernier moment et avec le vainqueur ». Or au moment de la débâcle serbe d'octobre 1915, il fut fait mention à plusieurs reprises de l'intérêt crucial de la Roumanie pour la réussite du front de Salonique : par ce pays se ferait la jonction entre le front de Salonique et la Galicie où l'allié russe était en train de se battre ardemment. En Grèce, le roi Constantin était pro-allemand, et le Premier ministre Venizelos pouvait tout juste accorder quelques gardes pour le camp fortifié qui se construisait à Salonique à partir de novembre 1915. Le $3^{\circ}$ bureau remarqua que les soldats grecs maintiendraient longtemps vis-à-vis des Français une « attitude douteuse ${ }^{13}$ et alla même jusqu'à craindre que «l'hostilité de la Grèce, qui a

10 SHAT, 16 N 3056, GQG Armées de 1'Est, $3^{\circ}$ bureau (1915-16), pièce n² 2, (7 octobre 1915).

11 SHAT, 16 N 3058, GQG Armées de 1'Est, $3^{\circ}$ bureau (1916-17), pièce nº 7, (6 novembre 1916).

12 SHAT, 16 N 3056, GQG Armées de l'Est, $3^{\circ}$ bureau (1915-16), note n¹0, (27 octobre 1915).

13 SHAT, 16 N 3060, GQG Armées de l’Est, 3º bureau (1917-18), dossier n², pièce $n^{\circ} 64,(11$ novembre 1917$)$. 


\section{ALEXIS TROUDE}

déjà hypothéqué toutes nos opérations dans les Balkans, ne les fasse pas définitivement échouer $»^{14}$.

Dernier facteur géopolitique, le rôle des autres grandes puissances. Les responsables politiques britanniques, relayés par les officiers de l'armée royale, freinaient les initiatives de la France. Lord Kitchener, Secrétaire aux Affaires étrangères, avait évoqué en novembre 1915 l'éventualité de faire retirer les troupes britanniques ; lorsque le général Sarrail prit le commandement en chef de l'Armée d'Orient en novembre 1916, les Britanniques maintinrent leur autonomie et, en pleine bataille de Monastir, refusèrent de renvoyer des renforts. En fait, l'Armée d'Orient abandonna à partir de 1917 la zone d'influence du Vardar et de Salonique aux Italiens. Ceux-ci renforcèrent également leur présence chez leur protégé albanais : l'armée italienne pénétra ainsi en 1917 à El-Basan « sans les Français et sans Essad Pacha $»^{15}$. Selon les accords de Londres d'août 1915, l'Italie devait récupérer la Dalmatie et l'Istrie dans l'Adriatique (= barrage aux prétentions serbes sur l'Adriatique $)^{16}$.

Enfin, il nous faut mentionner une implantation économique française en Serbie débutée au tournant du siècle et qui s'accéléra à l'approche du conflit mondial. La « banque FrancoSerbe, banque d'affaires créée au tournant du siècle à Paris, avait investi 60 millions de francs dès 1902 dans les chemins de fer serbes et 100 millions deux ans plus tard dans l'équipement militaire serbe ${ }^{17}$. La France avait des participations dans les mines de charbon de Bor et Negotin, en Serbie orientale, mais aussi dans les mines de fer de Trepča et de cuivre de Leskovac en Vieille-Serbie; Manufrance avait fourni dans les années 1910 l'armée serbe en fusils-mitrailleurs et en canons de $75^{18}$. Enfin, le réseau ferré serbe était largement la réalisation de constructeurs français : l'axe Belgrade-Salonique par Uskub en Macédoine venait juste d'être terminé lorsque la guerre débuta, et on travaillait sur le projet Belgrade-Sarajevo ${ }^{19}$.

14 SHAT, 16 N 3058, GQG Armées de 1'Est, $3^{\circ}$ bureau (1916-17), pièce n7, (6 novembre 1916).

15 SHAT, 16 N 3058, GQG Armées de 1'Est, $3^{\circ}$ bureau (1916-17), pièce n²1.

16 Le Moal, F. (2004) Le poids des ambitions adriatiques de l'Italie sur les opérations militaires dans les Balkans 1914-18, Cahiers du CEHD, n²2, pp. $45-63$.

17 Jaksic, G. (1980) Les relations franco-serbes aux XIX XXX $^{\circ}$ siècles», Actes du Colloque des Langues Orientales d'avril 1980.

18 Lambour, A. (1971) Politique des fournitures d'armes de la France en Europe centrale en 1900-1914, Paris.

19 Entretien avec Ljiljana Mirkovic, Directrice des Archives de Serbie, Belgrade, avril 1990. Sur les infrastructures et les intérets français dans les mines. 


\section{ALEXIS TROUDE}

\section{L'armée française en 1915 : soutien et rapprochement avec la Serbie}

En 1914, la vaillance des Serbes contre les Puissances centrales commençait à être connue en France et provoqua des vocations. Les correspondants du « Petit Journal », de l' «Illustration » et du «Parisien » rapportaient quotidiennement les faits et gestes de l'épopée serbe. Les Serbes avaient repoussé les Austro-Hongrois sur la Kolubara en août 1914, face à un ennemi six fois supérieur en nombre, et repris Belgrade. Or quelques volontaires français s'étaient déjà fait remarquer pour la défense de Belgrade.

\section{1-Janvier-novembre 1915 : missions médicale et aérienne françaises}

Pendant les combats que les Serbes menèrent seuls contre les Allemands et les Austro-Hongrois, deux missions militaires françaises allaient en 1915 venir aider l'armée serbe. Déjà Belgrade était défendue par trois canons de 140 et quelques dizaines de civils - la mission D-, et la frégate du lieutenant Picot défendait l'embouchure de la Save et du Danube ${ }^{20}$. Pas moins de 1200 tirailleurs-marins, aviateurs, artilleurs ou télégraphistes allaient ainsi, avant la formation de l'Armée d'Orient, rentrer en contact avec la population et l'armée serbe ${ }^{21}$.

D'avril à août 1915, une mission formée de plus de 100 médecins officia à Belgrade, avec comme tâche principale de lutter contre les épidémies qui commençaient à se propager. L'épidémie de typhus faisait rage et en mars 1915, déjà 125 médecins serbes sur 300 étaient décédés. Etablie dans les hôpitaux de Niš et de Belgrade, et assistée d'infirmières britanniques, la mission française réussira en quelques mois à faire passer de 35 à $4 \%$ le taux de mortalité typhique. Des tournées de vaccination, des comités d'hygiène avec création de dispensaires, mais aussi un effort d'information, avec causeries, soupes populaires et actions explicatives dans les écoles de village, amenèrent ce résultat formidable ${ }^{22}$.

20 SHM, SS Z 35, dossier H3-Affaires serbes, Note du lieutenant Picot (attaché militaire), (15 février 1916); SHM, SS Z 35, dossier H3-Affaires serbes, Note d'Auguste Boppe (Ambassadeur de France), (23 février 1916).

21 Stojancevic, V. (1990) Les Français en Serbie en 1915, in: Rapports francoyougoslaves, Institut d'Histoire, Belgrade, pp. 174-181.

22 "Mission militaire médicale française en Serbie ", in: Revue francomacédonienne, n², mai 1916. Cette revue avait été publiée d'avril 1915 à décembre 1917 à Salonique, non loin des zones occupées par l'Armée d'Orient. Regroupant des articles d'officiers et de sous-officiers de l'Armée d'Orient, la Revue franco-macédonienne cherchait à illustrer le travail humanitaire et les œuvres sociales de cette armée (écoles, hôpitaux, etc), 
En janvier 1915, arriva à Niš une mission militaire formée de 80 soldats, 8 officiers-aviateurs et 8 avions, sous le commandement du major Vitraud. Ses objectifs consistaient à bombarder les positions ennemies, défendre le territoire serbe et enfin surveiller les mouvements allemands et austro-hongrois, notamment en Syrmie et au Banat. Les avions de type Farman avaient beaucoup soutenu l'armée serbe même si les Allemands en abattirent deux. L'escadrille française fut d'abord déplacée dans le village de Ralje, dans les environs de Belgrade, puis à Kraljevo. Les six derniers avions français ramenèrent en novembre 1915 des enfants et des femmes serbes en France ${ }^{23}$.

Les Belgradois se sentirent véritablement protégés par cette aide maritime, terrestre et aérienne française et déjà en 1915 nacquit une amitié entre soldats français et civils serbes. L'ambassadeur de France à Belgrade, Auguste Boppe, constatait le 23 février 1915 : « la mission D a été très appréciée en Serbie $»^{24}$ et « l'excellente organisation des missions françaises produit une impression profonde ; le contraste avec les missions d'artillerie russe et britannique est sensible ». Le major commandant la mission médicale française soulignait aussi les liens qui se nouèrent entre Français et Serbes au tout début du conflit. " Accueilli cordialement dans tous les milieux serbes, c'est surtout au contact du paysan, véritable force de la Serbie, que le médecin serbe put pénétrer et comprendre les qualités foncières de la race. Altruisme, amour du sol natal, culte fervent de la patrie, souci de l'honneur, telles sont les vertus capitales du Serbe; et ceci suffit pour expliquer l'attirance faite d'affinités électives qu'exerce sur nous cette race qu'une fraternité de cœur et non un vil calcul d'intérêt pousse vers la France et que nous devons, dans ces cruelles épreuves, aimer et assister fraternellement $\rangle^{25}$.

\section{2-Octobre-décembre 1915 : le sauvetage à Durazzo et la retraite de Corfou}

Après l'échec des Dardanelles à l'été 1915, une partie du corps expéditionnaire franco-britannique fut ramenée dans le port grec de Salonique. Le haut-commandement français comptait sur la combattivité des Serbes pour qu'ils retiennent assez longtemps les Austro-allemands le temps de préparer le contact

mais aussi à accoutumer les soldats de l'Armée d'Orient à cette terre de Macédoine en vue d'une installation à plus long terme.

23 Muzet, A. (1917) Le monde balkanique, chapitre « La défense de Belgrade », Paris: Flammarion.

24 SHM, SS Z 35, dossier H3-Affaires serbes, Note d'Auguste Boppe (Ambassadeur de France), (23 février 1916).

25 Médecin-major J-C, « La mission médicale française en Serbie », in Revue franco-macédonienne, $\mathrm{n}^{\circ} 2$, (mai 1916). 
avec l'armée russe en Galicie. Mais le 5 octobre 1915, soit le jour même du débarquement de la $156^{\circ}$ division d'infanterie à Salonique, les Bulgares ajoutaient deux armées à la $\mathrm{IX}^{\circ}$ armée allemande et à la $\mathrm{III}^{\circ}$ armée austro-hongroise ; le 9 octobre, les Austro-Hongrois prenaient Belgrade et avant même que la mission Bailloud n'ait franchi la vallée du Vardar, les Bulgares occupaient Skoplje. Dans sa note du 11 novembre 1915, le $3^{\circ}$ bureau constata : " l'armée serbe n'a pas présenté la force de résistance dont nous la croyions capable $»^{26}$. Mais en aucun cas « il ne faut abandonner l'armée serbe $»^{27}$, ne serait-ce que pour des raisons morales; mais aussi « afin d'éviter que l'Allemagne ne mette la main sur Salonique $»^{28}$.

Le 25 novembre 1915 fut donné l'ordre historique de retraite de l'armée serbe par le roi Pierre $\mathrm{I}^{\circ}$, qui refusait la capitulation. Commença alors un épisode tragique qui se terminera seulement le 15 janvier 1916 : la traversée de l'armée et de la cour royale serbes à travers les montagnes d'Albanie. Assaillie par le froid et les maladies, un tiers de l'armée serbe périra. Alors que la mission navale française affirmait dès le 25 octobre qu' " il est indispensable pour l'armée serbe qu'elle puisse continuer à subsister $»^{29}$, les Italiens se plaignaient dès le $1^{\circ}$ décembre d'être les seuls à prendre des risques. Le 20 décembre, alors que les premiers soldats serbes en haillons rejoignaient les ports de Valona et Durazzo, le lieutenant Gauchet se plaignit du frein à l'aide émis par les Italiens et prévint : « Les Serbes vont mourir de faim $»^{30}$. Le lieutenant-colonel Broussaud signalait l' " épuisement physique et moral complet » et des " coups de fusils des comitadjis albanais »; il évoqua aussi la mort de jeunes recrues par centaines le long des routes ${ }^{31}$. Or ce fut l'armée française qui, sur 120000 soldats serbes arrivés à pied sur la côte albanaise, en récupéra 90000 pour les transférer sur l'île grecque de Corfou.

Entre le 15 janvier et le 20 février 1916 furent ainsi évacués à Corfou plus de 135000 soldats serbes. Lorsqu'ils débarquèrent

26 SHAT, 16 N 3056, GQG Armées de 1'Est, $3^{\circ}$ bureau (1915-16), note n¹9, (11 novembre 1915).

27 SHAT, 16 N 3056, GQG Armées de 1'Est, $3^{\circ}$ bureau (1915-16), note n²0, (11 novembre 1915).

28 SHAT, 16 N 3056, GQG Armées de 1’Est, 3ºureau (1915-16), note n³, (12 octobre 1915).

29 SHM, SS Z 35, dossier H3-Affaires serbes, Télégramme du Commandant Laurens, (25 octobre 1915).

30 SHM, SS Z 35, dossier H3-Affaires serbes, Note du Lieutenant Gauchet, (20 décembre 1915).

31 SHM, SS Z 35, dossier H3-Affaires serbes, Note du Lieutenant-colonel Broussaud, (22 décembre 1916). 


\section{ALEXIS TROUDE}

sur l'île grecque, on pouvait lire dans le carnet de route du $6^{\circ}$ chasseurs alpins que "l'état d'épuisement des malheureux soldats serbes est extrême : il en mourait 40 par jour $»^{32}$. A Corfou, les médecins allaient entièrement rétablir cette armée en guenilles et les instructeurs la remettre sur pieds : deux hôpitaux militaires furent dès lors installés et fin mars plus aucune épidémie n'était à l'œuvre. Les Serbes étaient pour la première fois en contact avec des unités constituées de Français ordinaires - pas des aviateurs comme en 1915 - et qui n'avaient pas été préparés à cette aventure. Svetozar Aleksić, paysan du centre de Serbie, fut réjoui d'avoir été, durant le transport de Corfou, rasé, lavé et habillé comme de neuf. "Qu'ils (les Français) bénissent leur mère-patrie, la France. Ils nous ont alors sauvé la vie $»^{33}$. La même reconnaissance se retrouve dans la lettre du Ministre serbe de la guerre au général Mondésir, responsable de l'évacuation de Corfou. Le 24 avril 1916, il affirmait que « les chasseurs, pendant leur séjour à Corfou, ont gagné les cœurs des soldats et de leurs chefs par leur dévouement inlassable envers leurs camarades serbes $»^{34}$. Ce dévouement explique que «les Français portaient à leurs camarades serbes leurs équipements et leur donnaient la plus grande partie de leur pain $»^{35}$. De plus, les Français si proches et attentionnés avaient créé des liens indéfectibles. Le prince Alexandre dit en avril 1916 à Auguste Boppe : «Les Serbes savent aujourd'hui ce qu'est la France. Jusqu'ici, ils ne connaissaient que la Russie. Or nul part ils n'ont vu les Russes, partout ils ont trouvé des Français : à Salonique pour leur tendre la main, en Albanie pour les accueillir, à Corfou pour les sauver $»^{36}$.

\section{II- L'Armée d'Orient 1916-1917 : échanges et conflits}

\section{A-Les enjeux militaires du Front de Salonique}

\section{Un camp retranché loin du front de l'Ouest}

Le 5 octobre 1915 , la $156^{\circ} \mathrm{DI}$ britannique, directement arrivée des Dardanelles, débarquait dans le port de Salonique. A la fin du

32 SHAT, 16 N 3057, GQG Armées de 1'Est, $3^{\circ}$ bureau (1916-17), note 14, (5 février 1916).

33 Témoignage de Svetozar Aleksic, in: Paunic-Djordjevic, (1988) Tri sile pritisle Srbijicu (Trois puissances ont encerclé la petite Serbie), Belgrade, pp. 8-12.

34 Zivanovic, M. (1966) Sur l'évacuation de l'armée serbe de l'Albanie et sa réorganisation à Corfou (1915-1916), d'après les documents français, Revue historique (Belgrade) n ${ }^{\circ} \mathrm{XIV}-\mathrm{XV}$, Institut d'Histoire, Belgrade, p. 2.

35 Ibid.

36 Ibid, p 4. 
mois arrivèrent du front occidental pour les épauler la $57^{\circ} \mathrm{DI}$ et la $122^{\circ} \mathrm{DI}$ françaises. Parties vers le nord à Velez rejoindre l'armée serbe, elles furent stoppées par les troupes bulgares entrées en guerre le 5 octobre. En réponse, une aide militaire française assez importante fut acheminée dans la région : à Durazzo, 1700 wagons de farine de blé, à Corfou, 75000 fusils et 18 batteries de 75, enfin à Salonique, 24 canons de 155 et 3 batteries de $65^{37}$. Mais très vite les Français durent se replier sur Salonique et y construire un fort retranché, entouré par des massifs avoisinant les 2500 mètres d'altitude. Avec les Britanniques, l'Armée Française d'Orient allait tracer une ligne de fortification allant du village de Seres au nord-est de Salonique jusqu'au lac de Prespa sur la frontière albanaise.

A partir d'avril 1916, l'Armée Française d'Orient formée des Britanniques et des Français accueillit l'armée serbe, placée au coeur du dispositif. Le front restait relativement stable, hormis des percées comme celle de Monastir en novembre 1916 et de Pogradec fin 1917. Pourtant, quatre nouvelles divisions furent envoyées en décembre 1916, mais des désaccords entre le général Sarrail, commandant de ce qui devient l'Armée d'Orient fin 1916, et le général Milne, commandant les troupes britanniques, empêchèrent de relayer les ordres et expliquèrent les échecs, notamment dans le secteur de Florina. Alors que l'armée serbe était placée sous le commandement direct de Sarrail, les Britanniques s'abstenaient à partir de l'hiver $1916 \mathrm{de}$ participer aux opérations : l'attaque déclenchée en mai 1917 dans le secteur de Doïran fut un échec, par mauvaise synchronisation des attaques et dispersion des moyens ${ }^{38}$.

L'autre raison des échecs réside dans la conception même du front de Salonique. Jusqu'en 1918, ce front était considéré comme un appui aux Russes qui combattaient en Galicie. Le gouvernement français attendait l'entrée en guerre de la Roumanie, à laquelle elle proposa même des territoires de Transylvanie et du Banat. Mais l'entrée dans l'Entente de la Roumanie en août 1916 ne fit qu'éloigner tout espoir d'une prochaine jonction des fronts de Salonique et de Galicie. En effet, dès septembre 1916, les Roumains étaient défaits et occupés par les Austro-allemands ${ }^{39}$.

37 SHM, SS Z 35, Dossier H3 : « ravitaillement armée serbe ».

38 Lieutenant-colonel Gérard Fassy, « Le commandement unique aux armées alliées d'Orient », in Revue historique des armées, $\mathrm{n}^{\circ} 1$, 2002, pp 47-58.

39 Pingaud, C. (1921) Histoire diplomatique de la France pendant la Grande Guerre, tome 1, Paris. 


\section{2- Mésententes et incompréhensions entre Serbes et Français}

Au début de l'année 1917, la démoralisation gagna les troupes et les premières divisions se firent jour au sein de l'Armée d'Orient. Tout d'abord, les officiers serbes, déçus par les atermoiements du Haut commandement à l'automne et l'hiver 1916, semblaient remettre en cause la stratégie militaire des généraux français. Dans son rapport de février 1918, l'officier de liaison Strauss remarquait une "subordination mal définie " des officiers serbes face au Haut commandement. Strauss critiquait aussi l'esprit mercantile des Serbes, qui ne pensaient qu'à « faire du commerce et traiter $»^{40}$. En fait, ce qui gênait le commandement français, c'était la faiblesse momentanée des troupes serbes, alors qu'au début du conflit la France fondait toute son action dans les Balkans sur cette armée. Dans un rapport du 9 septembre 1917 , le $2^{\circ}$ bureau affirmait que l'armée serbe « ne présente plus qu'une valeur défensive restreinte » et que « son concours aux opérations de l'Armée d'Orient est devenu faible au début de l'année $1917 \aleph^{41}$. Il faut savoir que la Serbie s'était battue pendant un an contre un ennemi -l'Autriche-Hongrie- 8 fois supérieur en nombre; en second lieu, comme il était dit dans le rapport du 9 septembre 1917, cette armée fournit « un effort considérable » à l'automne 1916, ce qui avait « porté un coup sérieux à sa valeur militaire $\aleph^{42}$. Enfin, des divisions importantes entre le gouvernement serbe et certains exilés politiques commençaient à apparaître sur l'après-guerre.

$\mathrm{Au}$ sein même de l'armée française existait un bataillon « bosniaque », qui très vite posera problème à la hiérarchie militaire. Constitué en mai 1916, ce bataillon comptait des Monténégrins et des Serbes d'Herzégovine, qui s'étaient battus jusque là avec l'armée serbe ou bien s'étaient engagés dans l'armée du roi Nicolas du Monténégro. Ces deux armées ayant été vaincues en 1915, 1700 hommes seront incorporés pendant six mois dans l'Armée Française d'Orient ${ }^{43}$. Très vite des cas d'indiscipline ou d'insubordination apparurent, et à la dissolution du bataillon par Sarrail en sept $1916^{44}, 7$ bombes et plusieurs

40 SHAT, 16 N 3060, GQG Armées de 1'Est, $3^{\circ}$ bureau (1917-18), dossier 2, note $\mathrm{n}^{\circ} 49$, (24 février 1918).

41 SHAT, 16 N 3060, GQG Armées de 1'Est, $3^{\circ}$ bureau (1917-18), dossier 1, note $\mathrm{n}^{\circ} 7,(6$ septembre 1917$)$

42 SHAT, Ibid.

43 Lieutenant Bourlet, M. (2002) Les Slaves du Sud dans l'armée française pendant la Première Guerre mondiale, in: Revue historique des armées, $\mathrm{n}^{\circ} 1$ (La France et les Balkans), pp. 59-70.

44 SHAT, 6 N 236, Note $n^{\circ} 225$ de la $113^{\circ}$ brigade, (30 novembre 1916). 
armes furent trouvés dans le bateau qui les amenait vers le camp de prisonniers de Cervione en Corse. En fait, ces hommes étaient le plus souvent de fervents partisans de l'indépendance du Monténégro et, le roi ayant accepté d'abdiquer en faveur de la dynastie serbe des Karadjordjević, des dissensions fortes avec les Serbes amenèrent les autorités françaises à les isoler du Front de Salonique. La France appuyant en 1916 les négociations dans la perspective de créer un Etat slave du sud formé autour de la Serbie, les Monténégrins se trouvaient en porte-à-faux.

\section{Découvertes réciproques}

\section{Fraternité d'armes et reconnaissance des Serbes}

Au début du front de Salonique, soldats serbes et français se jaugaient car la vision de l'autre était difficile : les Etats-majors n'avaient pas préparé leurs soldats à une cohabitation et aucune explication des cultures autochtones n'avait été faite auprès des poilus d'Orient. Radenko Ivić, arrivant à Salonique en avril 1916, expliquait ainsi sa peur des Français. " Nous avons été mis en garde à vue et le bateau qui nous accueillait était empli d'hommes en armes qui nous défiguraient; le bateau était inondé de lumière venant de grands projecteurs ». Mihajlo Milojević se souvient aussi quel accueil il a reçu à Bizerte au printemps 1916 : « Les Français nous attendaient sur des chevaux, le revolver au ceinturon, la carabine au flanc et le sabre à la main $»^{45}$.

Or les Français expliquaient leur attitude suspicieuse, voir craintive : "Nous avions entendu parler de vous (=les Serbes) comme de sauvages qui veulent fuir. Mais quand vous avez montré que personne n'essayait de fuir, notre peur a disparu $\gg^{46}$. Cette incrédulité et cette peur du côté français provenaient d'une méconnaissance totale des peuples balkaniques par le poilu d'Orient à son arrivée en Macédoine. Le lieutenant Maurice Tetenoir, dans son journal de guerre, expliquait bien le long temps d'acclimatation pour ces soldats brutalement jetés dans une guerre qui se déroulait loin de leur patrie. Arrivé le 26 septembre 1915 sur le front de Salonique et placé dans le secteur de Kereves, Tetenoir reconnût d'abord avoir peu de contacts avec la population locale. Ainsi le 8 octobre 1915 : « Nous partons au camp installé à $4 \mathrm{~km}$. La pluie tombe à torrents, nous traversons la ville arme sur l'épaule. La population nous regarde ; les soldats et les officiers grecs nous dévisagent ». Le lieutenant savait que l'armée française allait devoir se battre aux côtés de l'armée serbe, mais il ne la connaissait pas encore.

45 Témoignage de Mihajlo Milojevic, in> Paunic-Djordjevic, op cit., pp 78-82. 46 SHAT, 16 N 3060, GQG Armées de 1'Est, $3^{\circ}$ bureau (1917-18), dossier 1, note $n^{\circ} 6$, (5 septembre 1917). 
Ainsi toujours le 8 octobre, Tetenoir écrivait : « La $176^{\circ}$ était partie à 11 heures pour embarquer à destination de la Serbie. Arrivés à la gare, contre-ordre ; il ne peut partir pour raison diplomatique. Le train venu de Serbie repart vide......Quelle est notre situation ici ? ». Sa mission était donc peu claire : aider des Serbes qu'il n'avait pas encore vus. Or même lorsqu'il dut les accueillir, Tetenoir avait peur de ne pas les reconnaître. Le 19 octobre, parti avec un peloton occuper le village de Gradec incendié par les Bulgares, le lieutenant dira : " Devant nous des Serbes qui paraît-il vont se replier cette nuit. Comment les reconnaîtrons-nous ?» Malheureusement, le lieutenant Tetenoir mourait trois jours après dans une embuscade, sans avoir connu les soldats serbes ${ }^{47}$.

Mais la proximité des garnisons - les Serbes furent placés au centre du dispositif de l'Armée d'Orient- et surtout la fraternité d'armes à partir de la percée du Kaïmaktchalan en septembre 1916 allaient vite aider à se connaître et s'apprécier.

Un rapport établi par l'officier de liaison à Corfou en mars 1917 semble indiquer que les soldats français s'entendaient bien avec ceux des autres nationalités. Notamment, les liens entretenus avec les Serbes étaient " très cordiaux et suivis $»^{48}$. Un an plus tard, l'officier de liaison Strauss auprès de l'armée serbe, confirmait le sentiment d'un fort rapprochement des poilus d'Orient avec leurs homologues serbes. Il dit ainsi : " Parfaitement accueillis en France et à Bizerte, lors des séjours de convalescence qu'ils y ont fait, les soldats serbes ont pour la France un sentiment marqué de reconnaissance ${ }^{49}$. Ce sentiment des officiers de liaison français est corroboré par les remarques et écrits d'aprèsguerre des intéressés, les soldats serbes. Ranko Aleksandrović raconte son voyage de Valona à Corfou : « Les Français nous ont accueillis comme des frères; ce sont des mères pour nous, je ne sais comment décrire combien ils nous ont sauvé à Corfou $»^{50}$. Radojica Petrović accomplit un parcours impressionnant pour rejoindre le front. Il est parti de Londres, puis a embarqué à Toulon pour Bizerte en juin 1917 avant de se retrouver sur le front de Salonique à l'été 1917. Le plus important pour lui était que les Français aient montré tout de suite un fort sentiment de solidarité : «Je remercie les Français de nous avoir fourni

47 Recueil de lettres du lieutenant Maurice Tetenoir, de la $176^{\circ}$ DIC, publié par le « Courrier du Président » de l' "Association des Poilus d'Orient et Anciens combattants », Paris, $\mathrm{n}^{\circ} 4$, (septembre 2001).

48 SHAT, 17 N 725, Dossier n², Document n 1103.

49 SHAT, 16 N 3060, GQG Armées de 1'Est, $3^{\circ}$ bureau (1917-18), dossier n², pièce $n^{\circ} 49,24$ (février 1918).

50 Témoignage de Ranko Aleksandrovic, in: Paunic-Djordjevic, op. cit, pp. $32-35$ 
des bouées pour le sauvetage à Bizerte, avant même de nous donner des vêtements $\|^{51}$. Mais c'est Danilo Kuzmić qui le mieux parviendra à décrire le sentiment d'amitié qui nacquit à Salonique entre Serbes et Français. A son arrivée à Salonique, il nota que « Les Français étaient joyeux de nous voir et nous joyeux d'être revenus $»^{52}$.

\section{Soutien des intellectuels à la cause serbe}

En fait, les slavisants de renom multiplièrent seulement au milieu de la guerre les conférences et ainsi faire connaître les peuples balkaniques. L'historien Ernest Denis publia son livre célèbre sur « La Serbie » en 1915 et Victor Bérard en 1916, c'est-àdire au moment où l'on prit conscience de l'aide militaire que cet Etat pouvait rendre à la France. Eux-mêmes peu au fait des particularités balkaniques au début de leur carrière, ces deux historiens réagirent à l'annexion de la Bosnie-Herzégovine par les Autrichiens en 1908 et commencèrent à partir de cette année une réflexion sur le rôle de la Serbie. Et puis les journalistes spécialisés allaient mieux faire connaître les réalités serbes. Henri Barby, correspondant de guerre au Journal, écrivit en 1915 une série d'articles sur les batailles menées à Kumanovo et à Bregalnitza pendant les guerres balkaniques. Charles Diehl, dans son ouvrage de vulgarisation «L'héroïque Serbie » qui parut en février 1915, relatait les victoires serbes à Tser et Kolubara ${ }^{53}$.

Auguste Albert était mitrailleur sur le front de Salonique. Il reviendra en Yougoslavie après la guerre où il se maria; il s'installa ensuite à Skoplje où il créa une usine d'huile avec son beau-père. Auguste Albert était étonné par l'amour du Serbe pour sa terre. Lorsqu'il se battait contre les Bulgares, le Serbe criait : «C'est ma terre, ne l'oublie pas ». Puis Auguste Albert ajoutait : «Dans l'offensive attendue depuis longtemps (=la percée du front) j'ai été frappé par des choses étonnantes. J'ai remarqué comment le soldat serbe s'agenouille sur son sol natal et l'embrasse. Ses yeux sont pleins de larmes et je l'entends dire : « ma terre $»^{54}$. Il faut savoir que les Bulgares avaient occupé de 1915 à 1918 tout le sud-est de la Serbie : leurs revendications portaient sur la Macédoine du Pirin et en Serbie sur les districts de Pirot, Leskovac et Vranje.

51 Témoignage de Radojica Petrovic, in: Paunic-Djordjevic, op. cit, pp. 45-48.

52 Témoignage de Danilo Kuzmic, in: Paunic-Djordjevic, op. cit, pp. 64-67.

53 Pavlovic, M. (1988) Témoignages français sur les Serbes et la Serbie 1912 1918, Belgrade: Narodna Knjiga.

54 Témoignage d'Auguste Albert, in: Djuric, A. (1986) Ovako je bilo : Solunci govore (C'était comme ça à Salonique-témoignages d'Anciens combattants), Belgrade. 
Les conférences en Sorbonne par de grands slavistes devenaient plus fréquentes en 1916. Emile Haumant et Victor Bérard, qui avaient créé le " Comité Franco-serbe », y développaient leurs idées généreuses sur la Serbie. En Sorbonne se tinrent aussi des manifestations réunissant universitaires, hommes de lettres et responsables politiques. L'historien Ernest Denis prononcera, rien qu'en 1916, pas moins de trois conférences sur les Serbes et la Yougoslavie ${ }^{55}$ : le 27 janvier 1916, le président de la République, Raymond Poincaré, y assista. Le 8 février 1917, 1' "Effort serbe » fut organisé par le comité l' «Effort de la France et ses alliés » : cette initiative permit d'envoyer plus de 67000 vêtements aux sinistrés en 1916. Enfin le gouvernement organisa, le 25 mars 1915 et le 28 juin 1916, des « Journées franco-serbes » dans toutes les écoles pour faire connaître notre allié lointain ${ }^{56}$.

Dans le prolongement de cette action, un élan de solidarité se manifestait en faveur des enfants touchés par la guerre. Plus de 1900 enfants serbes avaient ainsi trouvé refuge pendant la guerre en France. La retraite d'Albanie et l'occupation de la Serbie fin 1915 avaient beaucoup ému la population et ce furent des associations, comme celle des « Orphelins de guerre », qui les premières accueillirent ces enfants démunis. On les retrouva ensuite au lycée de Bastia, à Saint-Etienne comme à Viriville, donc dans toutes les régions de France. La solidarité nationale fonctionna à plein régime pour aider ces civils serbes : 1,5 millions de francs d'aide furent votés à l'été 1916 au Parlement et des fonds « serbes » allaient même être créés dans quelques villes. Enfin, plus de mille étudiants vinrent se former dans les universités françaises ; dans les années vingt, cinquante viendront chaque année ${ }^{57}$.

\section{8-20 : Libération de la Serbie et action politique de la France}

\section{La percée du front de Salonique en septembre 1918}

\section{La victoire décisive : accord parfait franco-serbe}

En septembre 1918, les colonnes du Général Tranié et du Maréchal Franchet d'Esperey perçaient le front de Salonique

\footnotetext{
55 Denis, E. (16 janvier 1916) La Serbie héroïque, in: Foi et vie, cahier B,. Lire aussi son ouvrage majeur sur la question serbe, La Grande Serbie, Paris.

56 Jaksic, G. (1940) Livre sur la France, Belgrade. Consulter aussi aux Archives de Serbie (Belgrade), les pièces de l'Exposition « Français et Yougoslaves 1838-1988 » organisée à Belgrade en 1988.

57 Torau-Bayle, M. (1920) Réorganisation de l'armée serbe et trahison de la Grèce, in Salonique, Monastir et Athènes, Chiron, Paris.
} 
dans le massif de la Moglena et, en l'espace de trois semaines, libéraient la Macédoine et la Serbie. Le général allemand Mackensen déclarait lors de cet événement : « Nous avons perdu la guerre à Salonique ».

Ces opérations militaires menées ensemble finirent de souder les liens entre Serbes et poilus d'Orient et de nouer une amitié indéfectible. Paul Roi, élève-officier dans l'artillerie, évoquait l'habitude des combats qui avait fini de rapprocher les deux armées. «La joie des Français et des Serbes dès le moment où les canons tonnent. Ces canons ont comme redonné espoir aux soldats serbes dans la pensée du retour proche dans leur patrie. Nous, Français, avions une patrie. Tous les soldats français étaient conscients de cette situation ; de là leur volonté de se battre épaule contre épaule pour la liberté de la terre serbe $\gg^{58}$. Georges Schweitzer, officier-artilleur à Monastir en 1916 puis à la Moglena en septembre 1918, racontait l'abnégation des soldats serbes pendant la bataille. Blessé et perdu dans une tranchée dans le massif de la Moglena, Schweitzer fut sauvé d'une mort assurée par plusieurs Serbes venu le soigner dans la tranchée. « D'un coup, j'ai compris que j'étais entouré d'amis, de gens fantastiques, des soldats serbes qui sont maintenant là, à côté de moi ». Les Bulgares continuèrent à s'approcher en lançant des grenades, mais sa peur avait disparu. "Mes blessures sont soignées, le sang ne coule plus mais ce qui est le plus important : je ne suis plus seul. C'est maintenant la lutte pour moi : quand un soldat serbe se relève et lance une bombe, il le fait pour moi, il défend ma vie ! ${ }^{59}$. Georges Schweitzer, dans une hallucination extatique, éprouvait toute sa reconnaissance à l'esprit de sacrifice et de corps des soldats serbes accourus pour le sauver. A ce moment-là de la guerre, la solidité des liens entre Serbes et Français expliquait en partie la victoire obtenue par Franchet d'Esperey.

La confiance fut telle à la fin de la guerre entre soldats serbes et français qu'on décela de véritables scènes de liesse et des fêtes mémorables dans les bivouacs de l'Armée d'Orient. Albert Chantel, officier de liaison à la Moglena en septembre 1918, racontait la joie des Serbes à la vue de troupes françaises. Un officier serbe passant à côté de lui avec son escouade au retour d'une mission de surveillance s'écria : « Ce sont des Français, des Français -francuzi ! ». Et les soldats serbes se mirent à danser et à chanter. " Ses soldats, heureux, riaient comme s'ils allaient à une fête ».

58 Paul Roi, in: Djuric, A. (1986) Ovako je bilo : Solunci govore (C'était comme ça à Salonique -témoignages d'Anciens combattants), Belgrade.

59 Georges Schweitzer, in: Djuric, A. (1986), op cit. 


\section{Accueil chaleureux de l'armée d'Orient en Serbie}

Lors de leur remontée à travers les vallées du Vardar et de la Morava, des scènes de liesse populaire accompagnèrent l'Armée d'Orient. Les civils serbes, qui avaient appris les hauts faits militaires de cette armée, furent reconnaissants de leur avoir rendu leur famille et libéré leurs territoires.

Le général Tranié, qui libéra Skoplje en Macédoine puis Djakovica et Mitrovica au Kosovo-Métochie, nous a laissé des témoignages saisissants de l'amour d'un peuple pour son libérateur. A Kuršumlija, sur la route qui menait de Mitrovica à Niš, « les gens sont habillés pauvrement, les enfants presque nus, mais la population nous offre ce qu'elle a, les maisons sont largement ouvertes aux Français $»^{60}$. Partout sur la route menant à Niš, des scènes d'accolade, des offrandes de pain, de vin et de fromage, toujours données de bon cœur par un peuple pourtant touché par la disette. Arrivés à Niš, la seconde ville serbe, les soldats de l'Armée d'Orient furent accueillis avec tous les honneurs : les plus vieux ne laissaient pas le général Tranié remonter à cheval et l'embrassaient comme s'il était leur fils. Puis en remontant la vallée de la Morava, des actes symboliques très forts, qui allaient sceller l'amitié franco-serbe, émaillaient le chemin. A Aleksinac, le général Tranié fut enthousiasmé par l'accueil qui lui fut réservé : « De jeunes filles chantent la Marseillaise et m'entraînent dans la ronde dansée par tout le village $»^{61}$. Plus loin, à Čuprija, le maire de la ville fit un discours en français et les soldats serbes offrirent en guise de cadeau à l'Armée d'Orient des foulards ; à Svilajnac, des demoiselles offrirent au général Tranié un drapeau brodé de lettres d'or par leurs mères où il fut écrit en lettres cyrilliques : "Aux libérateurs de la Serbie, les demoiselles de Resava! $»^{62}$.

\section{Implantation française 1919-20}

\section{Projet d'implantation durable en Macédoine}

Le dépouillement de la "Revue Franco-Macédonienne », journal des officiers de l'Armée d'Orient qui a publié plus de 15 numéros mensuels entre septembre 1916 et fin 1917, nous permet d'attester de la volonté française de s'établir durablement dans la région une fois la guerre terminée. On sait qu'autour de Goritza (Korçë) a été établie pendant plusieurs mois une République avec écoles, routes et droit français. En mars 1917,

60 Général Tranié, in: Djuric, A. (1978) Les soldats de Salonique parlent, Belgrade, p. 58.

61 Général Tranié, in: Djuric, A. (1978), op. cit., p. 64.

62 Ibid, p. 65. 
un article souligna ainsi l'importance de l'influence culturelle pour les responsables français. Domaine placé au-dessus de l'économie, dans lequel la France était considérée plus forte que les autres, la culture représentait « un produit (sic) où la concurrence nous sera la moins dangereuse $»^{63}$. C'est dans cet état d'esprit que fut construite par l'armée française l'école de Lembet en Macédoine. Accueillant 220 élèves, tous civils, sur une population de 4500 âmes, l'école française de Lembet dispensait des cours d'histoire et de géographie en langue grecque, mais les mathématiques et les « leçons de choses » se faisaient dans la langue de Voltaire. L'auteur de l'article écrit en mai 1917 dans la « Revue Franco-Macédonienne » émettait l'espoir que cette école « restera après la guerre comme un modèle de la culture française en Macédoine $\aleph^{64}$. Cette action se poursuivit après l'armistice : dans un compte-rendu envoyé au Quartier général le 10 février 1919, l’officier de liaison déclara qu'il « faudrait envoyer des publications de France car il n'est pas encore parvenu ni livres ni journaux français durant cette guerre $»^{65}$. Or dès octobre 1918, le gouvernement serbe lui-même avait demandé la création d'un journal en français à Skoplje.

Mais c'est surtout au niveau des infrastructures économiques que va porter à la fin de la guerre l'effort de la France. En 1917, le gouvernement français dépêcha en Macédoine hydrologues, géographes, historiens et linguistes. Dans un premier temps, ils allaient mettre en plan toute la région contrôlée par l'Armée d'Orient : la cartographie complète du pays réalisée par l'Armée d'Orient remplaça la « carte autrichienne incomplète et inexacte $»^{66}$. Ensuite, un réseau de routes quadrilla la Macédoine : une voie Kastoria-Salonique comprenant de nombreux ponts sur la Moglenitsa, et une route Florina-Velez, donc vers la Serbie, furent construites. Enfin, les ressources du sol et du sous-sol furent exploitées. L'Armée d'Orient assècha les marais autour de Kastoria et Verria pour en faire des cultures maraîchères. Des mines de charbon (Komotini) et des gisements de fer (Kavala) approvisionnèrent les industries de Salonique ${ }^{67}$.

Mais le plus étonnant fut le projet d'installer dans la région des combattants nord-africains après la guerre. Un article de

63 « La culture française en Macédoine », Revue Franco-Macédonienne, n8, (mars 1917).

64 « L'école française de Lembet », Revue Franco-Macédonienne, n9, (mai 1917).

65 SHAT, 20 N 522, dossier n8, Note du 10 (février 1919).

66 Ancel, J. (1921) Travaux et jours de l'Armée d'Orient, Paris: Brossard, pp. $78-79$.

67 Ibid. 
décembre 1917 de la "Revue Franco-Macédonienne » nous éclaire ainsi sur les espoirs caressés par les officiers de l'Armée d'Orient. La similitude entre l'Afrique du nord et la Macédoine était décrite dans ses aspects géographiques et sociaux, incitant le soldat à émigrer dans cette région balkanique. « Des rues étroites, pavées à l'arabe, bordées de petites maisons » donnent l'occasion à l'auteur de souligner que Macédoniens et Africains possédaient « les mêmes coutumes, les mêmes caractères et les mêmes mœurs ». D'ailleurs, la Macédoine « semble être résignée depuis des siècles à vivre sous le protectorat (sic) d'un autre pays plus grand » et les Macédoniens montraient de «petites ambitions de cultivateur et d'épicier ». L'Africain pouvait donc s'y installer sans y être dépaysé, d'autant plus que le vide que représentait " le nombre trop restreint de nos compatriotes » devait être comblé ${ }^{68}$.

\section{Politique active de la France}

L'Armée d'Orient n'arrêta pas de combattre en octobre 1918 avec la capitulation de la Bulgarie. En effet, alors que le général Tranié poursuivait son avancée jusqu'à Trieste dans l'Adriatique, l'autre partie des armées françaises s'installa à Constantza, avec pour mission de bloquer le nouvel ennemi, le bolchévique. Jusqu'en 1921 stationneront des soldats français dans l'embouchure du Danube, mais avec beaucoup d'atermoiements devant un ennemi souvent invisible, ce qui entraînera des formes de lassitude.

Le plus important pour notre sujet réside dans le fait que cette situation idéale sur le Danube va amener la France à jouer un grand rôle dans la construction du « Royaume des Serbes, Croates et Slovènes ». Les frontières de cet Etat créé en décembre 1918 étaient l'oeuvre de géographes français, comme Ernest Denis. Des juristes français furent très vite après la guerre envoyés dans ce nouvel Etat, ce qui explique que le système politique y ressemblait fortement. En effet, dans 1' Etat des Serbes, Croates et Slovènes, une Assemblée nationale de 315 députés élus pour 4 ans au suffrage universel direct et à la représentation proportionnelle fut formée dès 1919. L'administration fut aussi fortement imprégnée des valeurs françaises. Enfin, un système départemental avec 33 unités dirigées par des préfets fut installé, ce qui remplaçait les anciennes régions historiques.

Dans le domaine économique aussi, la politique de la France se fit sentir dès l'après-guerre. En Serbie, Lafarge exploita les mines de cuivre de Bor et le gisement de charbon de Rudnik ;

68 « La politique française en Orient », Revue Franco-Macédonienne, $\mathrm{n}^{\circ} 12$, (décembre 1917). 
en Bosnie, des entreprises françaises prenaient possession des mines de fer de Zenica et de charbon à Banja Luka ; enfin en Macédoine, le gisement de Prilep intéressa les Français. Sur les pas de l'Armée d'Orient, pour mieux desservir ces mines au départ, furent construites des routes qui allaient devenir des axes importants. Ainsi les voies Bor-Negotin et KratovoVranje furent construites au début des années vingt. A la même époque, la voie ferrée Belgrade-Sarajevo était terminée par des compagnies françaises.

\section{Conclusion}

La Première Guerre mondiale a permis à la France d'approfondir son implantation dans les Balkans. Sur les plans économique, politique et culturel, le travail entamé par l'Armée Française d'Orient puis l'Armée d'Orient pendant la guerre a permis à la France de se présenter comme puissance de premier plan dans les Balkans dans les années 1920. Grâce au soutien indéfectible à ses alliés serbe, grec et roumain, la France put remplacer les Puissances centrales dans les Balkans.

Cette politique d'intérêt créera les conditions favorables pour l'émergence d'une amitié indéfectible entre les peuples serbe et français. Encore dans les années 1932, un train entier de journalistes spécialistes mais aussi de simples citoyens fit le trajet Paris-Belgrade pour témoigner de l'attache profonde qui liait ces deux peuples. A Belgrade, le sculpteur Ivan Meštrović érigea un monument où il était écrit «Nous aimons la France comme elle nous a aimé » et en France beaucoup de nos villes se paraient de rues en référence à des personnalités ou des lieux de Serbie. Mais l'assassinat en 1934 du roi Alexandre Karadjordjević et du Président du Conseil Louis Barthou, et l'arrivée du communisme en 1943 en Yougoslavie amenuiseront cette flamme. Pourtant, le Général de Gaulle évoquera toujours la Serbie au lieu de parler de la Yougoslavie et encore dans les années 1980, une troupe de théâtre serbe itinérante, remontant le trajet de la colonne Tranié, était partout accueillie comme aux plus beaux jours de la libération de 1918 .

\section{BIBLIOGRAPHIE :}

Boppe, (1919) A la suite du gouvernement serbe, Brossard.

Fappa, (1921) Souvenirs d'un officier de liaison, Flammarion.

Francois, J. (1976) Uskub, c'est loin, Lavauzelle.

Labry, (1918) Avec le retrait de l'Armée serbe, Paris.

Sarrail, (1920) Mon commandement en Orient (1916-1918), Paris. 


\title{
ALEXIS TROUDE
}

\author{
Алексис Труд \\ Универзитет Versailles-Saint Quentin, Француска \\ ПРИЈАТЕЉСТВО ИЗМЕЪУ ФРАНЦУСКИХ И СРПСКИХ
ВОЈНИКА У ОКВИРУ ИСТОЧНЕ ВОЈСКЕ ИЗМЕЪУ \\ 1915. И 1918. ГОДИНЕ
}

\section{Сажетак}

Циљ нашег чланка је прочавање односа између Француске и Србије током Првог светског рата кроз анализу односа француских и српских војника. Већ 1903, када је династија Карађорђевића ступила на власт, спољна политика Србије се реоријентише ка Француској и напушта централне силе којима су били блиски Обреновићи. Француска у српском Пијемонту види занимљивог савезника у свом механизму заобилажења централних царстава. Већ у првим годинама двадесетог века, ова два фактора објашњавају знатно присуство француских предузећа у Србији. Путем инфраструктурних и банкарских пројеката, Француска почиње да се инсталира у Србији. Први светски рат биће прилика да се Француска заиста укотви на Балкану и створи нераскидиве везе са српским народом. Заједничке патње и братство по оружју сковано на Солунском фронту током три године (1916-1918) чврсто ће обележити приврженост Срба Француској. Српска војска, која је принуђена на повлачење пред Централним силама 1915, изразиће вечну захвалност Источној армији која након повлачења кроз Албанију у зиму 1915. спасава српске војнике и лечи их на острву Крф у пролеће 1916, и уз коју се потом, унутар једне изузетне јединице, бори све до пробијања Солунског фронта 15. септембра 1918. године. Победа код Доброг поља, захваљујући маршалу Франше д’Епереу и генералу Транијеу, биће прва одлучујућа победа Антанте. У Француској се истовремено одржавају предавања о Србији, у свим школама се организују француско-српски дани и збрињавање хиљада српске деце. Француска, најзад, током те три године развија привредне пројекте средњег рока у источној Европи, посебно у Македонији, чиме се шири њена трајна имплантација на истоку Европе. Ојачане везе олакшавају имплантацију Француске на Балкану у послератним годинама, стварањем Краљевине Срба, Хрвата и Словенаца у децембру 1918. године. Тиме што се опредељује за српску династију Карађорђевића, оцртавајући границе и постављајући централизовану администрацију, Француска Краљевину Срба, Хрвата и Словенаца претвара у свог највећег савезника у источној Европи. Тиме се у послератним годинама отвара пут ка појачавању војних, економских и културних веза између Француске и краљевине Карађорђевића.

Кључне речи: Династија Карађорђевића, Солунски фронт, Источна војска, утицај Франиуске, Србија 